\title{
A NOTE ON A THEOREM OF J. GLOBEVNIK
}

\author{
DMITRY KHAVINSON
}

In this note we offer a short proof of Globevnik's theorem [Gl1, Gl2] for simply connected domains. Yet, this idea alone is insufficient to give the proof of his result in its full generality in finitely connected domains [Gl2].

Theorem 1. Let $\mathbf{D}$ be the unit disk, $\mathbf{T}$ is the circle, $f \in C(\mathbf{T})$. If for any function $g$ in the disk-algebra $A:=A(\mathbf{D})$ such that $f+g \neq 0$ on $\mathbf{T} \Delta_{\mathbf{T}} \arg (f+g) \geq 0$, then $f$ extends analytically to $\mathbf{D}$.

\section{Proof.}

Assume that $f$ does not extend analytically to $\mathbf{D}$, then $\operatorname{dist}(f, A)=d>0$, where the distance is understood in the $C(\mathbf{T})$-metric. Of course, then for all rational functions $f_{n}$ uniformly approximating $f$ on $\mathbf{T}$ we can assume that $\operatorname{dist}\left(f_{n}, A\right)=$ $d_{n}>d / 2$. Let $g_{n}$ be the best $A(\mathbf{D})$ approximation to $f_{n}$. As is known from the rudiments of the theory of extremal problems as in, e.g., [Kh], [Du] (Chapter 8), $g_{n}$ is analytic across $\mathbf{T}$, and $\left|g_{n}-f_{n}\right|=d_{n}>d / 2>0$ everywhere on $\mathbf{T}$. Moreover, since $f_{n}$ is continuous on $\mathbf{T}$, there is an extremal function $F_{n}$ in the dual problem of finding

$$
\max \left|\int_{\mathbf{T}} f_{n} h d z\right|
$$

over all $h$ in the unit ball of the Hardy space $H^{1}$.

$F_{n}$ is also analytic across $\mathrm{T}$ and

$$
F_{n}\left(f_{n}-g_{n}\right) d z=\left|F_{n}\right| d_{n}|d z| \geq 0 \quad \text { on } \quad \mathbf{T} .
$$

Since $\Delta_{\mathbf{T}} \arg F_{n} \geq 0$ and $\Delta_{\mathbf{T}} \arg d z=2 \pi$, (1) implies that

$$
\Delta_{\mathbf{T}} \arg \left(f_{n}-g_{n}\right) \leq-2 \pi
$$

for all sufficiently large $n$.

The rest is standard. Assume that $f_{n}$ approximate $f$, so that $\left|f-f_{n}\right|<\varepsilon$ on $\mathbf{T}$ and $\left|f-g_{n}\right|=\left|f_{n}-g_{n}-\left(f_{n}-f\right)\right| \geq d_{n}-\varepsilon>0$ for all $\mathrm{n}$. Then,

$$
\Delta_{\mathbf{T}} \arg \left(f-g_{n}\right)=\Delta_{\mathbf{T}} \arg \left[\left(f_{n}-g_{n}\right)\left(1-\frac{f_{n}-f}{f_{n}-g_{n}}\right)\right]=\Delta_{\mathbf{T}} \arg \left(f_{n}-g_{n}\right) \leq-2 \pi,
$$

giving a desired contradiction.

Remarks. (i) The proof could still be shortened if one notices that the function $f_{n}-g_{n}$ is badly approximable, cf. [Ga] (p.177). Then, Poreda's theorem yields (2). (ii) This particular argument does not directly extend to multiply connected domains, the focus of the Globevnik's paper [Gl2]. There, $\Delta \arg d z=2-k, k$ being the number of the boundary components, and the latter number is $\leq 0$ for all $k \geq 2$.

The author gratefully acknowledges support by the National Science Foundation. 


\section{REFERENCES}

[Du] P. Duren, Theory of $H^{p}$ Spaces, Academic Press, 1970.

[Ga] J. Garnett, Bounded Analytic Functions, Academic Press, 1983.

[Gl1] J. Globevnik, Holomorphic extendibility and the argument principle,to appear in Complex Analysis and Dynamical Systems II, Proceedings of a conference held in honor of Professor Lawrence Zalcman's sixtieth birthday in Naharia, Israel, June 9-12,2003, Contemp. Math. [http://arxiv.org/abs/math/math.CV/0403446].

[Gl2] J. Globevnik, The argument principle and holomorphic extendibility, J. d'Analyse, to appear [http://arxiv.org/abs/math/math.CVCV/0405409].

[Kh] S.Ya. Khavinson, Two papers on extremal problems in complex analysis, AMS Translations, ser. 2, 1986, v. 129, 1-114

Department of Mathematical Sciences, University of Arkansas, Fayetteville, Arkansas 72701

E-mail address: dmitry@uark.edu 